\title{
Influence de la supplémentation du lait en magnésium sur la fermentation lactique réalisée par S. lactis et S. thermophilus
}

\author{
par
}

K.-S. AMOUZOU, H. PREVOST et C. DIVIES*

\section{Rés u m é}

La supplémentation du lait en magnésium (25 à $50 \mathrm{ppm}$ ) permet à la fois une stimulation globale de la fermentation lactique, mais également l'obtention d'un meilleur taux de survie des streptocoques lactiques. L'étude de la répartition du magnésium dans les différentes fractions protéiques du lait écrémé reconstitué, par la technique de filtration sur gel Sephadex G 50, en utilisant l'eau distillée comme éluant, a permis de montrer que le magnésium se répartit en trois zones : sous une forme « liée aux protéines " (27 à $30 \mathrm{ppm})$, une forme liée à la fraction peptidique $(8 \mathrm{ppm})$ et une forme libre $(64 \mathrm{ppm})$. L'incubation en présence de $S$. lactis et $S$. thermophilus déplace, dans nos conditions expérimentales, les équilibres de la répartition du magnésium observée. L'addition de magnésium a pour effet d'augmenter faiblement la teneur de celui-ci au sein de la fraction protéique jusqu'à une valeur maximale voisine de $37 \mathrm{ppm}$, ainsi une forte proportion reste sous forme ionique.

Mots clés

Lait - Fermentation lactique - Stimulation - Magnésium - Streptococcus thermophilus - Streptococcus lactis - Répartition du magnésium.

\section{S u m m a ry}

\section{INFLUENCE OF MILK MAGNESIUM SUPPLEMENTATION LACTIC FERMENTATION WITH S. LACTIC AND S. THERMOPHILUS}

Milk magnesium supplementation (25 to $50 \mathrm{ppm}$ ) permits both a general stimulation of lactic fermentation, and the obtention of a

\footnotetext{
Laboratoire de Microbiologie, Ecole Nationale Supérieure de Biologie Appliquée à la Nutrition et à l'Alimentation, Université de Dijon, Campus universitaire Montmuzard - 21100 Dijon.

* Auteur qui recevra la correspondance.
} 
better survival rate of lactic streptococci. The study of magnesium distribution in the different proteinic fraction of reconstituted skim milk, using the Sephadex G 50 chromatography method by elution with distilled water, allowed us to show that magnesium exists in three states: protein-bound (27 to $30 \mathrm{ppm})$, peptid-bound (8 p pm) and a free state $(64 \mathrm{ppm})$. Incubation with $\mathrm{S}$. lactis and $\mathrm{S}$. thermophilus transfers the balance of the magnesium distribution observed. One effect of adding magnesium is to increase its concentration among proteinic fraction to a maximal rate of $37 \mathrm{ppm}$, so that a high ratio remains in an ionic state.

\section{Key words}

Milk - Lactic fermentation - Stimulation - Magnesium - Streptococcus thermophilus - Streptococcus lactis - Magnesium distribution.

\section{INTRODUCTION}

En technologie fromagère, la vitesse de production d'acide lactique d'origine microbienne est primordiale. La conduite rationnelle de l'acidification doit donc s'affranchir des causes éventuelles de variations. Brulé (1974), a observé qu'il fallait prendre en considération la variabilité de la constitution minérale du lait. Les principaux facteurs responsables de variations de cette nature ont fait l'objet de nombreux travaux; (Kamal et coll., 1961; Felber, 1970; Miller 1970 ; Guegen 1971 ; Moore 1974 ; Roux et coll., 1974; Mouillet et coll., 1975). Il apparaît que la constitution minérale du lait varie sensiblement avec la race, l'alimentation, la productivité, le stade de lactation et l'environnement (sol, climat, saison). Plusieurs études font état de l'addition d'oligoéléments métalliques dans le lait. Ainsi, cet ajout peut entraîner une accélération de l'affinage des fromages à pâte cuite, (Hofi et coll., 1971). Saakjan et coll., (1982) soulignent que l'addition d'oligoéléments $(\mathrm{Mn}, \mathrm{Zn})$ active la maturation du gruyère et améliore sa qualité.

Dans une étude préliminaire de l'influence des éléments métalliques sur la cinétique de la fermentation lactique dans le lait, il nous est apparu que le magnésium, dans le cadre de sa variabilité naturelle, augmentait de façon significative la production d'acide lactique par des streptocoques.

Nous nous sommes donc plus précisément intéressés au magnésium dont la teneur dans le lait peut varier de 100 à 150 ppm suivant l'origine géographique (Veisseyre, 1975). Cet élément minéral forme facilement des complexes, comme cofacteur de la phosphorylation il est l'activateur d'un grand nombre de réactions enzymatiques du métabolisme microbien. Le magnésium, également nécessaire à la stabilisation des acides nucléiques (ARN et ADN), 
contribue au maintien de l'intégrité de la structure des ribosomes et des membranes cellulaires (Ebel et Gunther, 1980). Sa présence en quantité suffisante dans les milieux de culture est importante pour le bon déroulement des croissances cellulaires.

Nous présentons, dans cet article, l'effet du magnésium sur le déroulement de la fermentation lactique réalisée par deux souches de streptocoques d'utilité industrielle, Streptococcus lactis 144 C.N.R.Z et Streptococcus thermophilus 302 C.N.R.Z. Nous avons suivi par la technique de filtration sur gel, la répartition de cet élément au sein des différents constituants du lait incubé ou non.

\section{MATERIEL ET METHODES}

\section{Souches}

Streptococcus lactis 144 C.N.R.Z. et Streptococcus thermophilus 302 C.N.R.Z., proviennent de la collection du Centre National de Recherches Zootechniques (C.N.R.Z.) de Jouy-en-Josas.

Les cultures pures, en suspension dense dans du lait glycérolé à $0,8 \%$, sont conservées congelées à $-25^{\circ} \mathrm{C}$ en ampoules scellées. $\mathrm{Au}$ fur et à mesure des besoins, les cultures sont décongelées rapidement et repiquées dans du lait deux ou trois fois avant utilisation.

\section{Lait utilisé}

Nous avons utilisé du lait en poudre sec, écrémé, à solubilité instantanée, exempt d'antibiotique. Le lait est reconstitué dans de l'eau distillée déminéralisée, à raison de $120 \mathrm{~g} / 1$ puis stérilisé en flacons de $500 \mathrm{ml}, 10 \mathrm{~min}$ à $118^{\circ} \mathrm{C}$. Les flacons sont conservés à $4^{\circ} \mathrm{C}$ pendant $24 \mathrm{~h}$ au plus.

L'addition de magnésium sous forme de chlorure $\left(\mathrm{MgCl}_{2}, \mathrm{Ti}\right.$ trisol Merck) se fait après stérilisation afin d'éviter tout phénomène de précipitation précoce de la caséine. Les doses utilisées au cours de l'expérimentation représentent une variation du simple au double des quantités initialement présentes dans le lait.

\section{Dénombrements cellulaires}

Les streptocoques lactiques en croissance dans le lait forment des chaînettes plus ou moins longues et des amas d'agglutination. Afin d'obtenir une meilleure corrélation entre le nombre de colonies comptées sur milieu gélosé $\mathbf{M}_{17}$ (Shankar et Davies, 1977) et le nombre de cellules présentes dans l'échantillon, celui-ci est agité 2 min sur un agitateur à vibrations de type Vortex, avec comme milieu de dilution du tryptone-sel additionné de Tween 80 (1\%0). 
A la suite de cette opération, $73 \%$ des cellules sont sous forme isolée et $27 \%$ sous forme de courtes chaînettes. Nous avons défini un facteur de correction tenant compte de cette répartition cellulaire déterminé par observation microscopique au moment de l'ensemencement des boîtes de Pétri.

$$
\text { cf }=\frac{\Sigma \text { xi.nxi }}{100}
$$

xi : nombre de cellules par amas.

nxi : nombre d'amas à i cellule(s).

cf : nombre moyen de cellules générant une colonie.

Si nc est le nombre moyen de colonies comptées, le nombre de cellules $\mathrm{N}$ est donné par la relation :

$$
\mathrm{N}=\mathrm{nc} \times \mathrm{f} \times \mathrm{cf} \quad \mathrm{f}: \text { facteur de dilution }
$$

\section{Dosages enzymatiques}

Les dosages du lactose et du galactose sont effectués par méthodes enzymatiques selon les protocoles préconisés par Boehringer Mannheim (1980). Les échantillons de lait incubés sont déprotéinisés par l'acide trichloroacétique $1,2 \mathrm{M}$ puis neutralisés par la soude $1 \mathrm{~N}$. Le surnageant de centrifugation $(5000 \mathrm{~g}, 10 \mathrm{~min})$ est conservé à $-25^{\circ} \mathrm{C}$ jusqu'au moment du dosage.

\section{Filtration sur gel}

Les protéines du lait sont fractionnées sur colonne chromatographique Pharmacia de type K25/100, munie d'un collecteur de fraction FRAC 300 et d'un détecteur UV $(280 \mathrm{~nm})$ couplé à un enregistreur graphique. Le gel utilisé est du Sephadex G50 medium (Pharmacia Fine Chemicals) dont le domaine de fractionnement est compris entre 1500 et 30000 . Ce gel, préparé comme le préconise Lonnerdal (1980) est stabilisé par passage d'eau déminéralisée additionnée d'antibiotique (auréomycine $10 \mathrm{ppm}$ ) afin de limiter le développement des micro-organismes. L'homogénéité du gel est testée par passage de $0,5 \mathrm{ml}$ d'une solution de bleu dextran 2000 à $2 \%$ dans l'eau. La colonne est calibrée par un mélange de protéines témoins (Kit calibration Pharmacia, fig. 2). Les filtrations sont réalisées sur $2 \mathrm{ml}$ de lait incubé ou non avec l'eau distillée déminéralisée comme éluant. L'utilisation d'un tampon minéral a été proscrite, la mise au point du protocole expérimental ayant fait apparaître que sous l'action de la force ionique du tampon, les minéraux micellaires, notamment le magnésium, étaient immédiatement libérés, comme Yamasuchi et coll. (1967) l'ont déjà observé. 


\section{Dosage du magnésium}

Le dosage du magnésium s'effectue par spectrophotométrie d'absorption atomique (Unican S.P. 1900) selon les conditions opératoires préconisées par Linden (1971). Le dosage est opéré en présence de lanthane qui joue le rôle de tampon spectral (Pinta, 1979). Les conditions expérimentales sont les suivantes :

Longueur d'onde : $285,21 \mathrm{~nm}$.

Ouverture de la fente : 0,15 à $0,20 \mathrm{~mm}$.

Intensité de la lampe à cathode creuse : $4 \mathrm{~mA}$.

Flamme air : 0,5 bar.

Acétylène : 0,5 à 0,9 bar.

La précision du dosage est de $\pm 0,1 \mathrm{ppm}$, cependant des variations d'au plus $10 \%$ peuvent apparaître entre les résultats du dosage sur le total des fractions et le lait.

\section{Dosage des acides aminés}

Les amino-acides sont dosés selon la méthode de Moore et Stein (1954).

\section{RESULTATS}

\section{Influence du magnésium sur la viabilité des populations de streptocoques dans le lait}

Nous présentons, tableau 1, le nombre de colonies dénombrées sur le lait témoin $\left(\mathrm{E}_{1}\right)$ et sur le lait additionné de $50 \mathrm{ppm}$ de magnésium $\left(\mathrm{E}_{2}\right)$ respectivement pour Streptococcus lactis et Streptococcus thermophilus. Le nombre de colonies indiqué correspond à la moyenne du nombre compté $\left(\mathrm{n}^{\mathrm{C}}\right)$ sur 3 boîtes de Pétri, pour chaque échantillon. Nous avons mentionné sur le même tableau les différences constatées entre les 2 types de lait.

A partir de la formule

$$
\mathrm{N}_{2}=\mathrm{N}_{1} 2 \frac{\mathrm{l}}{\mathrm{tg}}\left(\mathrm{t}_{2}-\mathrm{t}_{1}\right) \mathrm{N}_{1} \text { et } \mathrm{N}_{2} \text { repré- }
$$

sentant respectivement le nombre de cellules $(N)$ au temps $t_{1}$ et $t_{2}$, il est possible de calculer le temps de génération ( $\mathrm{tg}$ ) entre chaque mesure. En effet, grâce au coefficient de correction, nous tenons compte de la répartition cellulaire sous forme d'amas au moment de l'ensemencement sur boîtes de Pétri. On constate qu'entre 0 et $6 \mathrm{~h}$, le temps de génération est diminué de 1 à $2 \mathrm{~min}$, pour les 2 souches; cependant, celui-ci est plus profondément modifié entre 6 et 8 h. Lors de cette phase de ralentissement de la croissance cellulaire, le temps de génération en présence de magnésium est diminué de $13 \mathrm{~min}$, pour Streptococcus lactis et de $12 \mathrm{~min}$, pour 
TABLEAU 1 - TABLE 1

Effet de l'addition de $50 \mathrm{ppm}$ de magnésium sur la viabilité des streptocoques dans le lait Effect of magnesium adding (50 ppm) on streptococci survival in milk

\begin{tabular}{|c|c|c|c|c|c|c|}
\hline \multirow{2}{*}{\multicolumn{2}{|c|}{ Temps d'incubation }} & \multirow[b]{2}{*}{ Essai } & \multicolumn{2}{|c|}{ Streptocacaus thermophieus $\left(43^{\circ} \mathrm{C}\right)$} & \multicolumn{2}{|c|}{ Streptococcus lactis $\left(32^{\circ} \mathrm{C}\right)$} \\
\hline & & & Nombre de colonies & $\begin{array}{l}\text { Différence du nombre de } \\
\text { colonies entre } E_{0} \text { et } E_{2}\end{array}$ & Nombre de colonies & $\begin{array}{l}\text { Différence dunombre de } \\
\text { colonies entre } E_{0} \text { et } E_{2}\end{array}$ \\
\hline & $\mathrm{Oh}$ & $\begin{array}{l}E_{0} \\
E_{2}\end{array}$ & $\begin{array}{l}28 \pm 2,2 \cdot 10^{5} \\
27 \pm 2,7 \cdot 10^{5} \\
\end{array}$ & & $\begin{array}{l}31,6 \pm 3 \cdot 10^{6} \\
31,6 \pm 3,1 \cdot 10^{6}\end{array}$ & - \\
\hline & $2 \mathrm{~h}$ & $\begin{array}{l}E_{0} \\
E_{2}\end{array}$ & $\begin{array}{l}28 \pm 2,8 \cdot 10^{6} \\
30 \pm 3 \cdot 10^{6}\end{array}$ & $2 \pm 3 \cdot 10^{6}$ & $\begin{array}{l}51 \pm 5,1 \cdot 10^{?} \\
55 \pm 5 \cdot 10^{?}\end{array}$ & $4,0 \pm 5,0 \cdot 10^{7}$ \\
\hline & $4 \mathrm{~h}$ & $\begin{array}{l}E_{0} \\
E_{2}\end{array}$ & $\begin{array}{l}31 \pm 3 \cdot 10^{7} \\
34 \pm 3,4 \cdot 10^{7} \\
\end{array}$ & $3 \pm 2,1 \cdot 10^{?}$ & $\begin{array}{l}32,5 \pm 3,5 \cdot 10^{8} \\
37,4 \pm 3,7 \cdot 10^{8}\end{array}$ & $4,9 \pm 3 \cdot 10^{8}$ \\
\hline & $6 \mathrm{~h}$ & $\begin{array}{l}E_{0} \\
E_{2}\end{array}$ & $\begin{array}{l}23 \pm 2,4 \cdot 10^{8} \\
26 \pm 2,6 \cdot 10^{8} \\
\end{array}$ & $3 \pm 2,6 \cdot 10^{8}$ & $\begin{array}{l}30 \pm 3 \cdot 10^{9} \\
36 \pm 3,6 \cdot 10^{9}\end{array}$ & $6 \pm 3,6 \cdot 10^{9}$ \\
\hline & $8 n$ & $\begin{array}{l}E_{0} \\
E_{2}\end{array}$ & $\begin{array}{ll}49 \pm 4 & \cdot 10^{8} \\
67 \pm 6 & \cdot 10^{8}\end{array}$ & $18 \pm 7 \cdot 10^{8}$ & $\begin{array}{l}46,8 \pm 4,6 \cdot 10^{9} \\
57,2 \pm 5 \cdot 10^{9}\end{array}$ & $10,4 \pm 5 \cdot 10^{9}$ \\
\hline & $12 h_{1}$ & $\begin{array}{l}E_{0} \\
E_{2}\end{array}$ & $\begin{array}{l}12 \pm 1,2 \cdot 10^{7} \\
24 \pm 2,4 \cdot 10^{7}\end{array}$ & $12 \pm 2,4 \cdot 10^{7}$ & $\begin{array}{l}47,6 \pm 4,7 \cdot 10^{8} \\
61,6 \pm 6 \cdot 10^{8}\end{array}$ & $14 \pm 6 \cdot 10^{8}$ \\
\hline & $24 \mathrm{~h}$ & $\begin{array}{l}E_{0} \\
E_{2}\end{array}$ & $\begin{array}{c}64 \pm 6 \cdot 10^{6} \\
104 \pm 10 \cdot 10^{6} \\
\end{array}$ & $40 \pm 10 \cdot 10^{6}$ & $\begin{array}{l}61 \pm 6 \cdot 10^{7} \\
73,2 \pm 7 \cdot 10^{7}\end{array}$ & $12,2 \pm 7 \cdot 10^{?}$ \\
\hline & $48 \mathrm{~h}$ & $\begin{array}{ll}E_{0} \\
E_{2}\end{array}$ & $\begin{array}{ll}20 \pm 2 & \cdot 10^{5} \\
34 \pm 3 & \cdot 10^{5} \\
\end{array}$ & $14 \pm 3 \cdot 10^{5}$ & $\begin{array}{l}39,1 \pm 3,1 \cdot 10^{6} \\
51 \pm 5,1 \cdot 10^{6}\end{array}$ & $11,8 \pm 5 \cdot 10^{6}$ \\
\hline & $72 \mathrm{~h}$ & $\begin{array}{l}E_{0} \\
E_{2}\end{array}$ & $\begin{array}{l}35 \pm 3,9 \cdot 10^{3} \\
49 \pm 4 \cdot 10^{3}\end{array}$ & $14 \pm 5 \cdot 10^{3}$ & $\begin{array}{l}38,2 \pm 3,8 \cdot 10^{3} \\
49,3 \pm 4 \cdot 10^{3}\end{array}$ & $11,1 \pm 4 \cdot 10^{3}$ \\
\hline & $96 \mathrm{~h}$ & $\begin{array}{l}E_{0} \\
E_{2}\end{array}$ & $\begin{array}{l}11 \pm 2 \cdot 10^{2} \\
14 \pm 1 \quad \cdot 10^{2}\end{array}$ & $3 \pm 1 \cdot 10^{2}$ & $\begin{array}{l}11 \pm 1,1 \cdot 10^{2} \\
16 \pm 1,6 \cdot 10^{2}\end{array}$ & $5 \pm 1,6 \cdot 10^{2}$ \\
\hline
\end{tabular}


Streptococcus thermophilus, si bien que le nombre de cellules viables en phase stationnaire est nettement plus élevé en présence de magnésium.

\section{Effets de l'addition de magnésium sur le déroulement de la fermentation lactique}

Nous avons représenté figure 1 l'évolution du lactose transformé et du galactose accumulé par Streptococcus thermophilus 302 C.N.R.Z., incubé dans du lait en présence de différentes concentra-

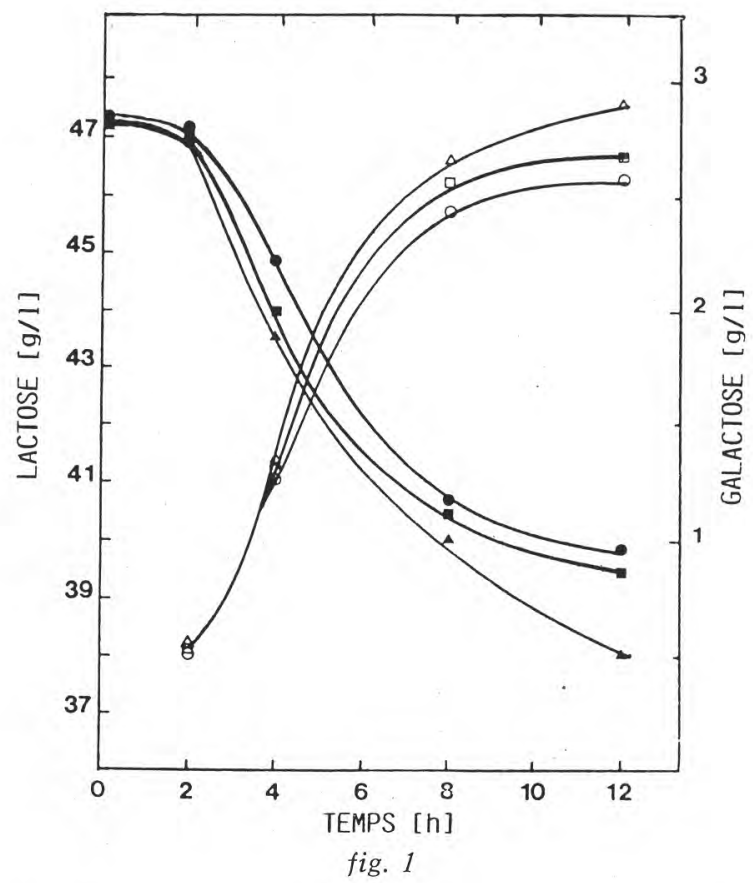

Evolution des teneurs en lactose et galactose dans le lait incubé en présence de S. thermophilus 302 CNRZ

Lait reconstitué

Lait reconstitué $+25 \mathrm{ppm}$ de $\mathrm{Mg}$

Lait reconstitué +50 ppm de $\mathrm{Mg}$

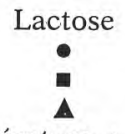

Galactose

Taux d'ensemencement : $2 \% \mathrm{v} / \mathrm{v}$ - Température : $43^{\circ} \mathrm{C}$.

Evolution of lactose and galactose concentration in milk incubed with S. thermophilus $302 \mathrm{CNRZ}$

Reconstitued milk

Reconstitued milk $+25 \mathrm{ppm}$ of $\mathrm{Mg}$

Reconstitued milk $+50 \mathrm{ppm}$ of $\mathrm{Mg}$

Inoculum : $2 \% v / v$ - Temperature : $43^{\circ} \mathrm{C}$.

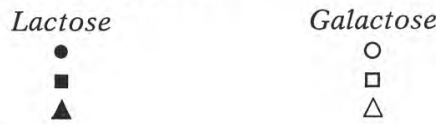


tions en magnésium. L'addition de 25 et 50 ppm de magnésium active la fermentation lactique. Nous avons vérifié que cette activation se traduit aussi bien dans l'utilisation du lactose par les bactéries que par la production d'acide lactique et l'accumulation de galactose. Afin de quantifier cette action du magnésium, nous avons reproduit, tableau 2 , les valeurs du pourcentage de lactose

\section{TABLEAU 2 - TABLE 2}

Pourcentage de lactose transformé, valeur relative du degré de lactose transformé et valeur relative de la population bactérienne obtenue dans le lait par addition supplémentaire de magnésium

Transformed lactose, relative transformation degree and relative data of the bacterial population obtains in milk after a supplementary addition of magnesium

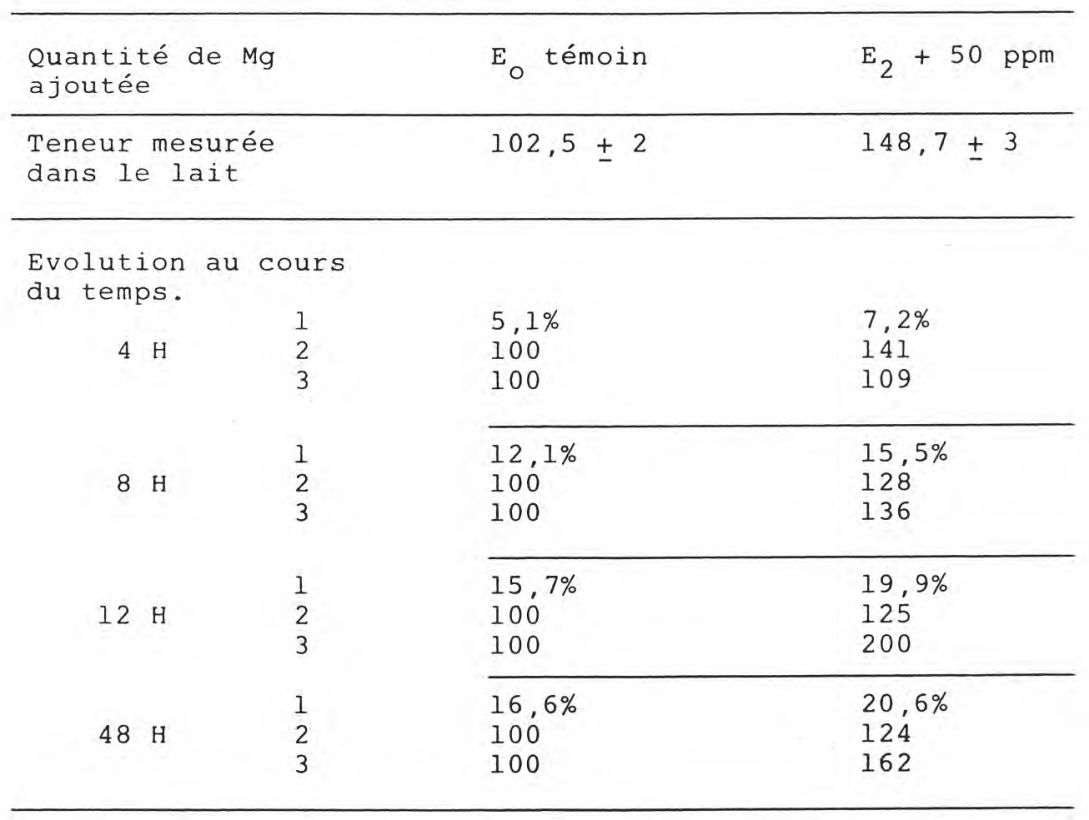

1. \% de lactose transformé initial

2. Valeur relative du degré de lactose transformé de l'essai $E_{2}$ par rapport au tēmoin $E_{0}$.

3. Valeur relative de la population de l'essai $E_{2}$ par rapport au témoin $E_{0}$. 
transformé dans le lait supplémenté ou non après $4,8,12$ et $48 \mathrm{~h}$ d'incubation. Ce tableau comporte également le degré relatif de lactose transformé et les valeurs relatives de la population bactérienne. On constate que l'action du magnésium est particulièrement notable sur la transformation du lactose durant les 4 premières heures d'incubation, alors que l'effet sur le nombre de cellules viables est très important au bout de $12 \mathrm{~h}$. Rappelons que la biomasse maximale est atteinte après $8 \mathrm{~h}$ d'incubation. Des résultats rigoureusement similaires ont été obtenus avec la souche de Streptococcus lactis 144 C.N.R.Z. Seulement 12,6\% du lactose transformé en $12 \mathrm{~h}$ au lieu de $15,7 \%$ pour Streptococcus thermophilus 302 C.N.R.Z. L'effet activateur est moins marqué. Comme il apparaît que l'effet du magnésium est particulièrement marquant au début de la fermentation, nous nous sommes attachés à identifier l'évolution de la répartition de cet ion durant les 4 premières heures d'incubation.

\section{Analyse de la répartition du magnésium parmi les constituants du lait}

Nous avons représenté, figure 2, l'analyse des 120 fractions obtenues pour notre matière première après la filtration sur gel. Pour le magnésium, on observe 2 pics importants.

Entre ceux-ci, la quantité de magnésium est faible mais ne s'annule jamais. L'analyse du chromatogramme permet de distinguer 3 zones. La zone I où le magnésium est lié aux protéines de poids moléculaire supérieur à 14000 , la zone II où le magnésium dit peptidique correspond aux molécules de faible poids moléculaire que sont les peptides et autres molécules protéiques libres, et enfin la zone III qui correspond au magnésium libre (ionique), on y retrouve conjointement les composés de faible poids moléculaire et les substances azotées non protéiques dialysables. Ainsi, en se référant aux 3 zones précédemment définies, nous avons représenté, tableau 3, les pourcentages de magnésium lié aux 3 classes de molécules en fonction du temps d'incubation et des doses de magnésium ajoutées. Le magnésium présent initialement dans le lait se trouve à $64 \%$ sous forme libre, la fraction liée aux protéines de poids moléculaire élevé est de l'ordre de $27 \%$ tandis que dans la zone II on ne retrouve que $8 \%$ du magnésium total. Cette répartition semble être modifiable selon, d'une part, l'addition de magnésium et, d'autre part, l'incubation du lait en présence de Streptococcus thermophilus. En effet, la fraction de magnésium lié aux molécules de la zone I passe de $27,3 \mathrm{ppm}$ pour le lait témoin à $36 \mathrm{ppm}$ pour le lait supplémenté de $50 \mathrm{ppm}$; cet enrichissement n'augmente pas pour des teneurs en magnésium plus élevées. L'incubation du lait témoin pendant $4 \mathrm{~h}$ en présence de Streptococcus thermophilus 302 C.N.R.Z. entraîne un déplacement de $10 \%$ de 


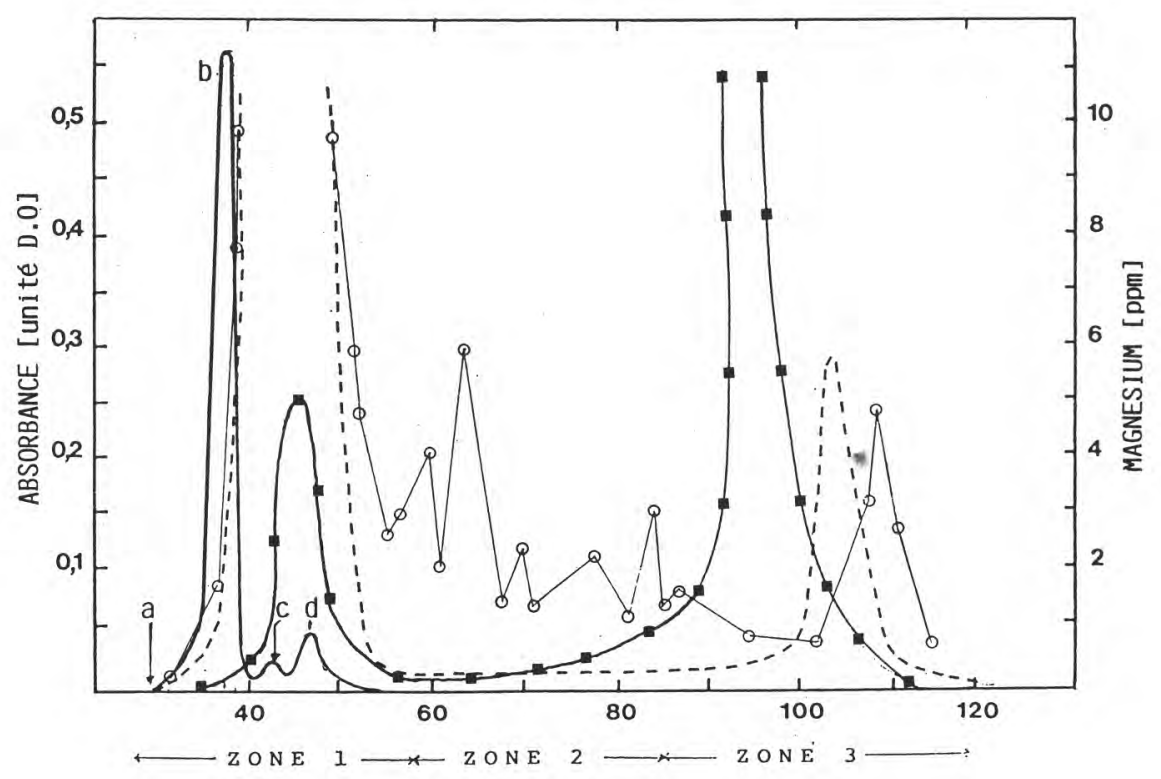

FRACTIONS

fig. 2

Filtration du lait reconstitué sur gel Séphadex G 50

Répartition de :

0 acides aminés $(570 \mathrm{~nm}) \quad$ - Magnésium (285 nm) - Protéines (280 nm).

a : Bleu Dextran 2000.

b : Phosphorilase b, ovalbumine.

c : Soybean Trypsin Inhibitor (STI). calibration

$\mathrm{d}: \alpha$-lactalbumine.

Eluant : eau distillée.

Volume des fractions : $5 \mathrm{ml}$.

Reconstitued milk filtration with Sephadex G 50

Distribution of :

0 Amino-acids - - Magnesium -- Proteins.

$a$ : Dextran blue 2000.

$b$ : Phosphorilase $b$, ovalbumin.

c: Soybean Trysin Inhibitor (STI).

d: $\alpha$-lactalbumin.

Elution with distilled water.

Volume of fractions : $5 \mathrm{ml}$.

magnésium libre vers les zones I et II, pour le lait supplémenté, le fait n'est pas noté par suite de la saturation en ions de la classe des molécules de la zone I. Ceci suggère qu'une partie importante du magnésium ajouté passe dans la fraction libre et reste sous forme ionique. Notons qu'en ce qui concerne Streptococcus lactis, nous avons mis en évidence les mêmes phénomènes. 
TABLEAU 3 - TABLE 3

Répartition du magnésium après filtration du lait incubé ou non en présence de Streptococcus thermophilus $302 \mathrm{CNRZ}$

Magnesium distribution after filtration of milk incubed with Streptococcus thermophilus 302 CNRZ

\begin{tabular}{|c|c|c|c|c|c|c|}
\hline Echantillon & $\begin{array}{l}\text { Temps } d^{\prime} \text { incubation } \\
43^{\circ} \mathrm{C}\end{array}$ & $\begin{array}{l}\text { Total de Mg dosé } \\
\text { directement dans } \\
\text { 1'échantillon (ppm) }\end{array}$ & $\begin{array}{l}\text { Total de Mg dosé } \\
\text { dans les fractions } \\
\text { (ppm) }\end{array}$ & $\begin{array}{l}\text { Zone I } \\
\text { Mgproteique }\end{array}$ & $\begin{array}{l}\text { Zone II } \\
\text { Mg peptidique }\end{array}$ & $\begin{array}{l}\text { Zone ift } \\
\text { Mg libre }\end{array}$ \\
\hline \multirow{2}{*}{$E_{0}$ ténoin } & Oh & 99,3 & 95,5 & 27.3 & 8,2 & 64 \\
\hline & $4 \mathrm{~h}$ & 103,6 & 103,2 & 35,1 & 16,3 & 51,8 \\
\hline$E_{2}$ & $\mathrm{Oh}$ & 150,6 & 147,6 & 36,3 & 17,3 & 94 \\
\hline$+50 \mathrm{ppm}$ & $4 \mathrm{~h}$ & 150,6 & 149,9 & 38,6 & 15,2 & 96,1 \\
\hline$E_{4}$ & $\mathrm{Oh}$ & 194,5 & 198,8 & 36,7 & 28,2 & 133,9 \\
\hline$+100 \mathrm{ppm}$ & $4 h$ & 194,5 . & 198,3 & 35,4 & 29,1 & 133,8 \\
\hline
\end{tabular}

\section{DISCUSSION - CONCLUSION}

De l'ensemble de nos résultats, il ressort que la supplémentation du lait en magnésium entraîne une activation de la fermentation lactique s'accompagnant d'une meilleure croissance des souches de streptocoques étudiées. Si bien que le nombre de cellules viables en phase stationnaire est plus élevé. Par contre, les cinétiques de mortalité cellulaire observées au delà de $8 \mathrm{~h}$ (tab. 1) ne sont pas modifiées.

Ces observations mériteraient, à notre avis, d'être expérimentées en technologie laitière, dans le but d'accélérer l'acidification de laits relativement carencés.

Examinons tout d'abord la répartition du magnésium parmi les constituants du lait. La technique de filtration que nous avons retenue pour suivre cette répartition respecte en grande partie les grandes classes de répartition de cet élément, puisque nous retrouvons dans le lait non enrichi 27 à $30 \mathrm{ppm}$ dans la zone micellaire. Brulé et Lenoir (1984) signalent que 25 à $30 \%$ de magnésium se retrouvent dans cette zone, soit sensiblement notre pourcentage, puisque le lait témoin contient $100 \mathrm{ppm}$ de magnésium. Le magnésium sous forme ionisé est de $64 \mathrm{ppm}$; le reste se répartit dans la zone dite "peptidique ". Dans les cas du lait supplémenté en magnésium, nous avons observé une augmentation de la proportion de cet élément au sein des micelles caséiniques de 30 à 37 ppm pour une addition de $50 \mathrm{ppm}$. Dans les gammes de concentrations 
plus élevées, cette teneur reste constante, ce qui suggère que les environnements micellaires sont rapidement saturés. Ainsi, la supplémentation du lait en magnésium à des teneurs supérieures à $50 \mathrm{ppm}$ a pour effet d'augmenter sa proportion sous forme ionique. Dans ces laits supplémentés en magnésium, l'incubation jusqu'à pH 5,4 en présence de Streptococcus lactis et Streptococcus thermophilus, ne modifie pas les équilibres (tab. 3). Par contre, dans les mêmes conditions, on observe dans les laits témoins la migration de $10 \%$ de magnésium ionisé vers les zones peptidiques [2] et micellaires [1]. La production microbienne d'acide lactique entraîne la solubilisation des sels minéraux, notamment du $\mathrm{Ca}^{++}$(Brulé, 1974). La faible diminution du magnésium ionique observée, reste inattendue. Ce fait pourrait s'expliquer, à notre avis, par la libération de peptides phosphorylés (zone II) piégeant du magnésium dans la zone peptidique, et par la fixation de magnésium au sein des micelles (zone I) sur les groupements de phosphate colloïdal.

En ce qui concerne la stimulation de la croissance cellulaire que nous avons observée, il semble que celle-ci soit liée à l'augmentation du taux de magnésium ionisé directement disponible pour les cellules. La magnésium intervient comme cofacteur d'un grand nombre de réactions enzymatiques, il participe à la structure des membranes cellulaires et des ribosomes (Paunier, 1974). L'influence des métaux dans la nutrition des bactéries lactiques a fait l'objet de peu de travaux. Cependant, il a déjà été montré que le magnésium et le fer pouvaient avoir un rôle dans la nutrition des streptocoques mésophiles (Reiter et Moller-Madsen, 1963; Ledesma et coll., 1977). Walker et Duffus (1980) ont mis en évidence leur rôle dans le contrôle de la division cellulaire de Schizosaccharomyces pombe et Kluyveromyces fragilis; le magnésium participerait au contrôle de l'assemblage des microtubules. Dans un article récent, Ates Saltukoglu et Slaughter (1983) ont signalé chez Saccharomyces cerevisiae qu'il était nécessaire de disposer dans le milieu d'une quantité de magnésium plus importante que nécessaire pour que la croissance cellulaire se déroule normalement, un excès de calcium aboutissant à une augmentation de la phase de latence et à un ralentissement de la vitesse de croissance. Dans le cas du lait, la présence excessive de calcium pourrait expliquer qu'on observe encore une activation du métabolisme et de la croissance des bactéries lactiques au delà de $60 \mathrm{ppm}$ de magnésium sous forme ionique. L'effet du magnésium sur la vitalité des cellules bactériennes a été abordé par de nombreux auteurs. Ainsi, Thomas et Batt $(1968,1969)$ ont montré chez Streptococcus lactis qu'en cas de carence d'autres sels minéraux, notamment les phosphates, l'absence de magnésium conduit à une perte de viabilité corrélée à une dégradation rapide de l'ARN. Dans nos conditions expérimentales (tab. 1), un tel phénomène n'a pas été observé puisque le taux de mortalité cellulaire n'est pas modifié. 


\section{Bibliographie}

Ates Saltukoglu ( ), Slaughter (J.C.) (1983). - The effect of magnesium and calcium on yeast growth. J. Inst. Brew., 89, 81-83.

BoEHringer (Mannheim) (1979). - Enzymatic analysis for food chemistry, 28 and 32.

BRULÉ (G.) (1974). - Les minéraux du lait. Revue Laitière Française, 400, 61-65.

Brulé (G.), Lenoir (J.) (1984). - La coagulation du lait. In « Le fromage », Ed. A. Eck, Lavoisier, Paris, 1-21.

EBel (H.), GuntheR (T.) (1980). - Magnesium metabolism. A review. J. Clin. Chim. Clin. Biochem., 18, 257.

Fel.BER (H.) (1970). - Zur labgerinnung der silage Milchteil II : mineral und eiweissgehalt von silage und micht-silage-milch. Die Osterreichische milchwirtschaft., 25 (2), 1-11.

GUÉGUEN (L.) (1971). - La composition minérale du lait et son adaptation aux besoins minéraux du jeune. Ann. Nutr. Alim., 25, A 335-A 381.

Hofi (A.A.), RifaI (I.D.), Salam (M.H.), Mahran (G.A.) (1971). - Utilisation des oligoéléments pour accélérer l'affinage des fromages à pâte dure. J. Dairy Res., 38, 203-204.

Kamal (T.H.), Johnson (H.D.), Ragsdale (A.L.) (1961). - Influence of the stade of lactation and environnemental temperature of the salt balance of milk. J. Dairy Sci., 44, 1655-1660.

Ledesma (O.V.), de Ruiz Holgano (A.P.), Olivier (I.G.), de Giori (G.S.), Raibaud (P.), GALPIN (J.V.) (1977). - A synthetic medium for comparative nutritional studies of lactabacilli. J. Appl. Bacteriol., 42, 123-133.

LiNDEN (G.) (1971). - Applications de la spectrophotométrie d'absorption atomique dans les laboratoires d'industrie alimentaire. Industr. Alim. agr., 88, 793-803.

LONNERDAL (B.) (1980). Chemical modification of dextran gels for gel filtration of trace elements ligands. Trace element analytical chemistry in med. and Biol. Walter de Gruyter and Co, Berlin, New York, 439-446.

Miller (M.) (1970), - Zn nutrition of cattle. J. Dairy Sci., 53 (8), 1123-1132.

Moore (S.) and Stein (W.H.) (1954). - J. Biol. Chem., 211, 907-913.

MooRE (J.) (1974). - Les métaux agents de pollution du lait, leur dosage par spectrophotométrie d'absorption atomique. Le Lait, 54, 139-152.

Mouillet (L.), Luquet (F.M.), Casalis (J.) (1975). - Contribution à l'étude des variations de la teneur en sels minéraux du lait de vache dans différentes régions française. Le Lait, 56, 683-694.

Paunier (L.) (1974). - Métabolisme du magnésium. Annale Nestlé, 67, 23-30. Ed. Services Scientifiques Nestlé.

Pinta (M.) (1979). - Spectrométrie d'absorption atomique. Application à l'analyse chimique, tome I et II, $2^{\mathrm{c}}$ édition, Lib. Masson, Paris.

Reiter (B.), Moller-Madsen (A.) (1963). - Cheese and butter starters. J. Dairy Res., 30, 419-456.

Roux (M.), Luguet (F.M.), Casalis (J.) (1974). - Sur la teneur en sels minéraux du lait de vache des régions du Nord et Sud-Ouest de la France. Ann. Fals. Exp. Chim., 67, 577-583.

SaAkJan (R.) (1982). - Utilisation d'éléments de trace Mn, Zn... en fabrication du Gruyère. Epev. Zoov. Inst., 3, 29-37. 
Shankar (P.A.), Davies (F.L.) (1977). - A note on the suppression of Lactobacillus bulgaricus in media containing $\beta$-glycerophosphate and application of such media selective to isolation of Streptococcus thermophilus from yoghurt. J. Society Dairy Technology, 30, 28-30.

Thomas (T.D.), BatT (R.D.) (1968). - Survival of Streptococcus lactis in starvation conditions. J. Gen. Microb., 50, 367-382.

VEISSEYRE (R.) (1975). - Technologie du lait, $3^{\text {e }}$ édition entièrement refondue de techniques laitières. Ed. La Maison Rustique, Paris.

Walker (G.M.), Duffus (J.H. (1980). - Magnesium ions and the control of the cell cycle in yeast. J. Cell. Sci., 42, 329-356.

YAMASUCHI (K.), HoNna (Y.), Tsugo (T.) (1967). - Separation of soluble calcium phosphate and citrate in milk by Sephadex gel filtration. Jpn J. Zootech. Sci., 38, 117-119. 